\title{
Cost Comparison of Urate-Lowering Therapies in Patients with Gout and Moderate-to-Severe Chronic Kidney Disease
}

\author{
Ghaith Mitri, MD; Eric T. Wittbrodt, PharmD, MPH; Robin S. Turpin, PhD; \\ Beni A. Tidwell, BS; and Kathy L. Schulman, MS
}

\begin{abstract}
BACKGROUND: Patients with chronic kidney disease (CKD) are at increased risk for developing gout and having refractory disease. Gout flare prevention relies heavily on urate-lowering therapies such as allopurinol and febuxostat, but clinical decision making in patients with moderate-tosevere CKD is complicated by significant comorbidity and the scarcity of real-world cost-effectiveness studies.
\end{abstract}

OBJECTIVE: To compare total and disease-specific health care expenditures by line of therapy in allopurinol and febuxostat initiators after diagnosis with gout and moderate-to-severe CKD.

METHODS: A retrospective observational cohort study was conducted to compare mean monthly health care cost (in 2012 U.S. dollars) among gout patients with CKD (stage 3 or 4 ) who initiated allopurinol or febuxostat. The primary outcome was total mean monthly health care expenditures, and the secondary outcome was disease-specific (gout, diabetes, renal, and cardiovascular disease [CVD]) expenditures. Gout patients (ICD-9-CM 274.xx) aged $\geq 18$ years with concurrent CKD (stage 3 or 4 ) were selected from the MarketScan databases (January 2009-June 2012) upon allopurinol or febuxostat initiation. Patients were followed until disenrollment, discontinuation of the qualifying study agent, or use of the alternate study agent. Patients initiating allopurinol were subsequently propensity score-matched (1:1) to patients initiating febuxostat. Five generalized linear models (GLMs) were developed, each controlling for propensity score, to identify the incremental costs (vs. allopurinol) associated with febuxostat initiation in first-line (without prior allopurinol exposure) and second-line (with prior allopurinol exposure) settings.

RESULTS: Propensity score matching yielded 2 cohorts, each with 1,486 patients (64.6\% male, mean [SD] age 67.4 [12.8] years). Post-match, $74.6 \%$ of patients had stage $3 \mathrm{CKD} ; 82.9 \%$ had CVD; and $42.1 \%$ had diabetes. The post-match sample was well balanced on numerous comorbidities and medication exposures with the following exception: $50.0 \%$ of febuxostat initiators were treated in the second-line setting; that is, they had baseline exposure to allopurinol, whereas only $4.2 \%$ of allopurinol initiators had baseline exposure to febuxostat. Unadjusted mean monthly cost was $\$ 1,490$ allopurinol and $\$ 1,525$ febuxostat $(P=0.809)$. GLM results suggest that first-line febuxostat users incurred significantly $(P=0.009)$ lower cost than allopurinol users $(\$ 1,299$ vs. $\$ 1,487)$, whereas second-line febuxostat initiators incurred significantly $(P=0.001)$ higher cost $(\$ 1,751$ vs. $\$ 1,487)$. Febuxostat initiators in both settings had significantly $(P<0.001)$ higher gout-specific cost, due to higher febuxostat acquisition cost. Increased gout-specific cost in the first-line febuxostat cohort was offset by significantly $(P<0.001)$ lower CVD (\$288 vs. $\$ 459)$ and renal-related cost (\$86 vs. \$216). There were no significant differences in either renal or CVD costs (adjusted) between allopurinol initiators treated almost exclusively in the first-line setting and second-line febuxostat patients.
CONCLUSIONS: Gout patients with concurrent CKD, initiating treatment with febuxostat in a first-line setting, incurred significantly less total cost than patients initiating allopurinol during the first exposure to each agent. Conversely, patients treated with second-line febuxostat following allopurinol incurred significantly higher total cost than patients initiating allopurinol. There was no significant difference in total cost between the agents across line of therapy. Although study findings suggest the potential for CVD and renal-related savings to offset febuxostat's higher acquisition cost in gout patients with moderate-to-severe CKD, this is the first such retrospective evaluation. Future research is warranted to both demonstrate the durability of study findings and to better elucidate the mechanism by which associated cost offsets occur.

\section{J Manag Care Spec Pharm. 2016;22(4):326-36}

Copyright $\odot 2016$, Academy of Managed Care Pharmacy. All rights reserved.

\section{What is already known about this subject}

Urate-lowering therapies (ULTs) such as allopurinol and febuxostat are the mainstay of treatment for gout flare prevention, and clinical trial results suggest that febuxostat is as effective as allopurinol in lowering serum uric acid levels.

For patients with moderate-to-severe chronic kidney disease (CKD), management of gout is complicated by significant comorbidity and potential hypersensitivity reactions to allopurinol, resulting in suboptimal dosing of allopurinol for some CKD patients.

Previous economic comparisons of allopurinol and febuxostat have compared overall patient populations in real-world settings, but no study has done so in a renally impaired population.

\section{What this study adds}

This study is the first to employ real-world data to compare economic outcomes among gout patients with preexisting stage 3 or 4 CKD initiating allopurinol or febuxostat.

This analysis, which employed propensity score matching to analyze a large administrative database, demonstrated that in the first-line setting, gout patients with concurrent CKD treated with febuxostat incurred significantly less total cost than patients treated with allopurinol, despite the higher drug acquisition cost of febuxostat.

These findings suggest that quantifying the cost burden associated with ULT may be more complex than a simple comparison of drug acquisition cost alone. 
G out, a metabolic disorder that causes acute, intermittent, and painful flares of arthritis in the joints of the foot, knee, hand, and wrist, occurs in some patients when there is a sudden onset of inflammation as a result of excess serum uric acid (sUA) in the blood (hyperuricemia) with deposition of crystals in tissue. It affects more than 3\% of the U.S. adult population. ${ }^{1}$ Gout prevalence is rising, in part due to an ever-increasing prevalence of comorbidities that promote hyperuricemia (e.g., hypertension, obesity, metabolic syndrome, type 2 diabetes mellitus, and chronic kidney disease [CKD]) and in part due to the extensive use of thiazide and loop diuretics for cardiovascular disease (CVD). ${ }^{2-4}$

The disease is more common in older adults, with an incidence of $8 \%$ in individuals aged 70-79 years compared with only $1.7 \%$ in those aged less than 50 years. ${ }^{5}$ Data from the Rochester Epidemiology Project suggest that the incidence of gout without diuretic exposure doubled in the United States between the 1970s and the 1990s. ${ }^{1}$ Studies have also described increased incidence of gout among populations with chronic renal failure ${ }^{1}$ and increased prevalence with successive stages of CKD. ${ }^{6}$ Among those with self-reported gout, $71 \%$ reported having CKD stage $\geq 2 .^{7}$ CKD has also been shown to be an independent risk factor for more refractory gout. ${ }^{8}$

Numerous studies have demonstrated the substantial economic burden associated with gout, ${ }^{9-16}$ with the annual direct burden of illness for new cases of acute gout among men in the United States estimated to be $\$ 27.4$ million. ${ }^{10}$ Gout has also been shown to have a substantial impact on a patient's healthrelated quality of life (HRQOL)., ${ }^{2,17-19}$ Gout symptoms can cause significant discomfort, disruption, and disability with the patient's HRQOL in the areas of sexual function, sleep, social life, emotional health, hobbies, and footwear. ${ }^{20}$ Both the physical and mental health consequences worsen as the frequency and severity of flares increase. ${ }^{21}$

Gout flare prevention relies heavily on urate-lowering therapies (ULTs) such as allopurinol and febuxostat, ${ }^{22}$ and a primary goal of treatment is to achieve a sUA level target of $<6 \mathrm{mg} / \mathrm{dl} .^{2}$ However, management of gout in renally impaired patients is multifaceted, as clinical decision making is complicated by significant additional comorbidity. Hypersensitivity reactions to allopurinol are more likely to occur in patients with renal impairment, for whom a reduced dose of allopurinol is recommended, although there is no precise dosing consensus., ${ }^{43}$ The failure of patients to reach target sUA and thus reduce gout flare risk has been associated with the appropriateness of allopurinol dosing, a low frequency of sUA measurements, and poor allopurinol compliance. ${ }^{24}$

Clinical trial results suggest that febuxostat $80 \mathrm{mg}$ per day is more effective than allopurinol $300 \mathrm{mg}$ per day in lowering sUA levels. ${ }^{25-27}$ However, some researchers have suggested that, given its comparatively high acquisition cost, use of this medication should be limited to patients with renal impair- ment, those intolerant to allopurinol, and those who do not achieve sUA targets on the maximum recommended dosage of allopurinol. ${ }^{28,29}$ However, few studies have compared the cost burden associated with specific gout therapies in real-world settings, and no study, to our knowledge, has done so in a renally impaired population.

The primary objective of this study was to estimate total and disease-specific cost among gout patients with preexisting stage 3 or 4 CKD who initiated allopurinol or febuxostat. The study hypothesis was that the higher acquisition cost of febuxostat would be offset by decreases in renal and CVD-specific costs due either to febuxostat's greater clinical effectiveness in lowering uric acid levels or to the underdosing of allopurinol in renally impaired patients. This hypothesis presumes a causal relationship between hyperuricemia and CVD outcomes, for which there is a growing body of evidence. ${ }^{30-36}$

\section{Methods}

The data source for this research was the MarketScan Commercial Claims and Medicare Supplemental/Coordination of Benefits databases. Patients were included in the analysis if they were aged $\geq 18$ years and had at least 31 days of continuous therapy (i.e., more than two 30-day prescriptions) with either allopurinol or febuxostat between January 1, 2009, and June 30, 2012. Patients were also required to have a diagnosis (International Classification of Diseases, Ninth Revision, Clinical Modification [ICD-9-CM]) for gout (274.xx) on a nondiagnostic claim either before or within 30 days of initiating either agent. In addition, patients were required to have a diagnosis of stage 3 or 4 CKD (ICD-9-CM 585.3 or 585.4) on at least 1 nondiagnostic claim either before or within 30 days of ULT initiation. Furthermore, patients were required to have at least 12 months of continuous eligibility before ULT initiation, at least 31 days after initiation, and complete data availability for the duration of their study eligibility. Patients with end-stage renal disease or evidence of transplant, dialysis, non-skin-based malignancy, or HIV/AIDS in the 12 months preceding the index date; that is, baseline, were excluded from the study. Patients were allowed to contribute only the initial episode of treatment with each agent to the study. Patients were followed, within each episode, until disenrollment from MarketScan, discontinuation of the qualifying ULT (defined as a gap in therapy greater than 30 days), or use of the alternate study agent. The databases complied with all aspects of the Health Insurance Portability and Accountability Act of 1996, and the study data were de-identified and therefore exempt from institutional review board approval.

Propensity score matching ${ }^{37-40}$ was employed to minimize the impact of selection bias. Patients treated with febuxostat were matched to patients treated with allopurinol with similar predicted probabilities using the nearest neighbor method, without replacement, and a caliper equal to one tenth of the standard error of the estimated propensity score. Explanatory 


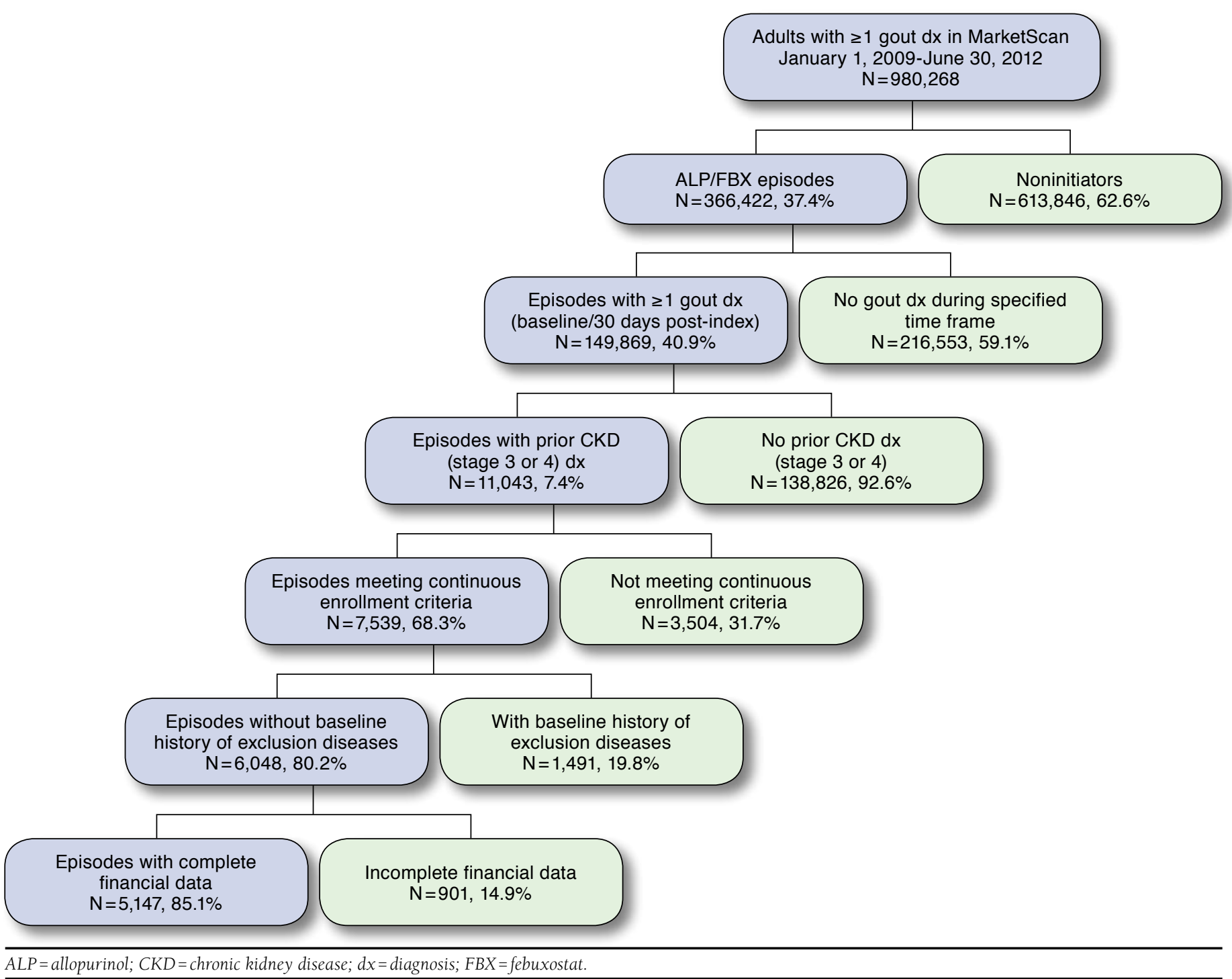

variables in the propensity model included basic demographics (age group, sex, U.S. census region, benefit design, index year); cardiovascular conditions and other comorbidities (baseline history of dysrhythmia/conduction disorder, heart failure, chronic liver disease, stroke, chronic obstructive pulmonary disease, rheumatoid arthritis, diabetes, dyslipidemia, or cellulitis); baseline medication exposure (antibiotics, opiates, angiotensin-converting enzyme [ACE] inhibitors, angiotensin II receptor blockers [ARBs]); and CKD stage nearest index as a measure of renal disease severity. Measures of gout severity in the model included baseline evidence of tophi, number of baseline gout flares, using an algorithm published by Kim et al. (2013) to define gout flares, ${ }^{41}$ and medications used to treat gout flares (colchicine, glucocorticoids, nonsteroidal antiinflammatory drugs [NSAIDs], or probenecid). As a measure of resource intensivity, the model included baseline cost per patient per month. Post-match balance across study covariates was considered acceptable if the standardized difference between the 2 cohorts was $<10 \%,{ }^{42}$ and traditional tests of significance (e.g., chi-square, t-test) were $>0.05$.

The primary study outcome was direct medical expenditure (health plan-insured paid amounts, coordination of benefits, patient copayment, deductible, and coinsurance amounts) as measured during each treatment episode. Secondary study outcomes included disease-specific cost per month. Gout-specific expenditure was defined as claims with a diagnosis of gout 
Cost Comparison of Urate-Lowering Therapies in Patients with Gout and Moderate-to-Severe Chronic Kidney Disease

TABLE 1 Baseline Demographic, Clinical, and Gout Characteristics Pre- and Post-Propensity Matching

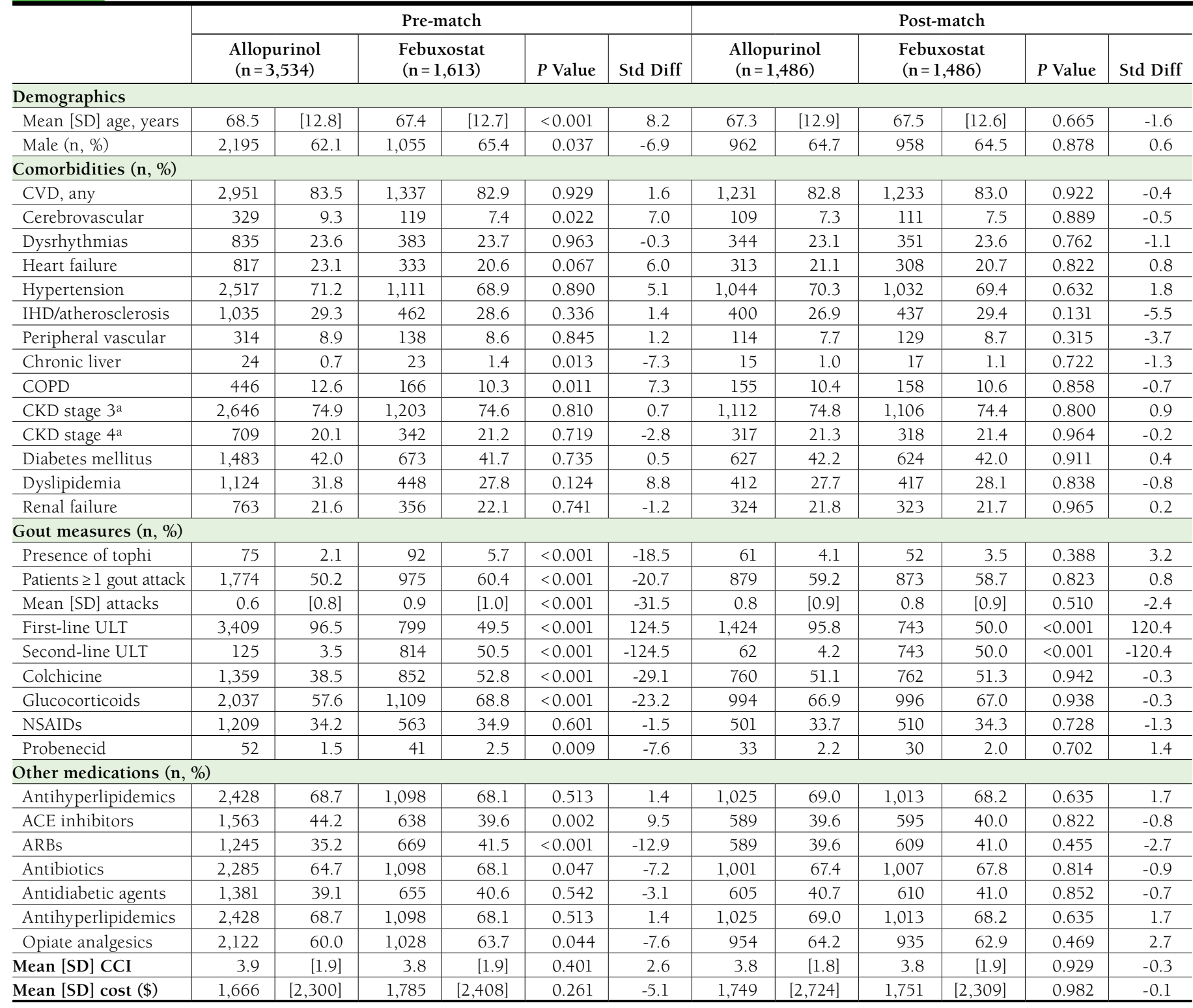

${ }^{a} \mathrm{CKD}$ stage based on the stage reported nearest the study index date.

$A C E$ inhibitor = angiotensin-converting enzyme inhibitor; $A R B=$ angiotensin II receptor blocker; $C C I=$ Charlson Comorbidity Index; $C K D=$ chronic kidney disease;

$C O P D=$ chronic obstructive pulmonary disease; $C V D=$ cardiovascular disease; $I H D=$ ischemic heart disease; NSAID = nonsteroidal anti-inflammatory drug; $S D=$ standard deviation; Std Diff=standard difference; ULT= urate-lowering therapy.

or any of the following gout-specific medications or services: allopurinol, colchicine, febuxostat, probenecid, glucocorticoids, prescription NSAIDs, sulfinpyrazone, intra-articular aspiration and/or injection, microscopic examination of specimen from musculoskeletal system/joint fluid, or uric acid measurement. Non-gout-specific expenditure was categorized into 4 mutually exclusive disease groups: renal, diabetes, cardiovascular, and other non-gout. The 4 non-gout disease groups were defined using a combination of ICD-9-CM diagnosis codes, specific medications, and/or medication classes (see the Appendix, available in online article). The "other non-gout" group was defined as the presence of any code that was not included in the definition of gout, renal, diabetes, or cardiovascular. The cost applied to each disease category was assigned proportionally based on the total number of diagnoses on the claim and the number of diagnoses associated with each disease group. 
TABLE 2 Cost Per Patient Per Month in the Post-match Sample, Unadjusted ${ }^{a}$

\begin{tabular}{|c|c|c|c|c|c|}
\hline & \multicolumn{2}{|c|}{ Allopurinol $(n=1,486)$} & \multicolumn{2}{|c|}{ Febuxostat $(n=1,486)$} & \multirow[b]{2}{*}{$P$ Value } \\
\hline & Mean, \$ & $\mathrm{SD}, \$$ & Mean, \$ & $\mathrm{SD}, \$$ & \\
\hline Total cost & 1,490 & 3,945 & 1,525 & 3,867 & 0.809 \\
\hline Gout-specific cost & 87 & 389 & 243 & 265 & $<0.001$ \\
\hline Non-gout-specific cost & 1,403 & 3,861 & 1,282 & 3,820 & 0.389 \\
\hline Renal & 214 & 1,293 & 171 & 1,693 & 0.435 \\
\hline Cardiovascular & 459 & 1,856 & 384 & 1,900 & 0.272 \\
\hline Diabetes & 182 & 974 & 165 & 652 & 0.581 \\
\hline Other & 548 & 930 & 562 & 880 & 0.668 \\
\hline
\end{tabular}

A sensitivity analysis was conducted, which assigned cost solely based on the primary diagnosis.

Multivariate analyses were employed because the propensity model could not adjust for the line of ULT treatment for the 2 cohorts. Five generalized linear models (GLMs) were developed, controlling for propensity score, to identify the incremental costs (total, gout, cardiovascular, diabetes, renal-related costs) associated with initiating febuxostat (vs. allopurinol) in first- and second-line settings. Total, diabetes, cardiovascular, and renal-related costs were modeled with gamma distributions and log link; gout-specific cost was modeled with a gaussian distribution and identity link. Distributional assumptions were determined by the Park test. Goodness-of-fit was assessed using scaled deviance and scaled Pearson metrics. Two part models were employed if the proportion of patients with nonzero cost exceeded $5 \%$.

\section{Results}

\section{Population Selection}

Among the 980,268 adults with a gout diagnosis in the MarketScan databases between January 1, 2009, and June 30, 2012, 37\% ( $n=366,422)$ had a prescription for allopurinol or febuxostat. After applying the remaining study criteria, a total of 5,147 episodes in 4,941 patients were entered into the propensity model. The most common reasons for exclusion were no history of stage 3 or $4 \mathrm{CKD}$ before allopurinol/febuxostat initiation, failure to meet continuous enrollment criteria, and prior use of allopurinol or febuxostat (Figure 1).

There were notable differences in the pre-match sample at baseline. Febuxostat patients were younger and more likely to be male, to reside in the southern United States, to have commercial insurance, and to have a history of chronic liver disease and prior exposure to ARBs, antibiotics, or opiate analgesics/agonists. Moreover, febuxostat patients were more likely to have tophi documented on a medical claim, to have had more frequent gout flares, and to have had prior exposure to allopurinol; that is, to have been treated with febuxostat in a second-line setting. Accordingly, febuxostat patients were also more likely to have received colchicine, glucocorticoids, or probenecid before initiation.

After propensity matching, the final post-match sample contained 1,486 episodes in each cohort. A total of 206 patients had an episode in both cohorts (6.9\%). The post-match sample was well balanced on all covariates except prior exposure to the alternate agent (Table 1). Fifty percent of febuxostat initiators had no prior exposure to allopurinol; that is, they were receiving first-line treatment as opposed to $95.8 \%$ of allopurinol patients. Mean (standard deviation [SD]) duration of follow-up was 8.6 (7.3) months in the febuxostat cohort and 8.7 (7.6) months in the allopurinol cohort. Eighty-three percent of the post-match sample had a history of CVD (hypertension [69.9\%], ischemic heart disease [28.2\%], dysrhythmia or other conduction disorder [23.4\%], or heart failure [20.9\%]). Forty-two percent had diabetes and, by definition, $100 \%$ had renal disease.

Mean (SD) dose per day of allopurinol during follow-up was 183.4 (94.1) $\mathrm{mg}$, with $25 \%$ of patients receiving a dosage of at least $260 \mathrm{mg}$. Mean (SD) dose per day of febuxostat during follow-up was 50.1 (17.8) $\mathrm{mg}$, with $25 \%$ of patients receiving a dosage of at least $62 \mathrm{mg}$. Patients in the allopurinol cohort were significantly more likely to receive colchicine during followup $(P=0.005)$ but were less likely to receive glucocorticoids $(P=0.025)$ than patients in the febuxostat cohort. During the follow-up period, 5.2\% of allopurinol patients and 5.0\% of febuxostat patients progressed to end-stage renal disease.

\section{Economic Outcomes}

Unadjusted Analyses. There were no significant differences in mean monthly per patient expenditure, unadjusted $(\$ 1,490$ allopurinol, \$1,525 febuxostat; Table 2). Gout-specific cost per month represented $10.9 \%$ of total cost. Cardiovascular, renal, diabetes, and other non-gout-specific costs represented, respectively, $31.4 \%, 14.3 \%, 12.9 \%$, and $41.3 \%$ of non-gout cost.

Overall, febuxostat patients were significantly less likely to have had an emergency room visit $(P=0.013)$, and they had fewer hospitalizations $(P=0.017)$. However, when hospitalized, febuxostat patients had significantly longer lengths of stay 
TABLE 3 Gout-Specific Cost and Utilization in the Post-match Sample, Unadjusted ${ }^{a}$

\begin{tabular}{|c|c|c|c|c|c|}
\hline & \multicolumn{2}{|c|}{$\begin{array}{c}\text { Allopurinol } \\
(n=1,486)\end{array}$} & \multicolumn{2}{|c|}{$\begin{array}{c}\text { Febuxostat } \\
(\mathrm{n}=1,486)\end{array}$} & $P$ Value \\
\hline \multicolumn{6}{|l|}{ Gout-specific utilization } \\
\hline Hospitalization, any (n, \%) & 62 & 4.2 & 58 & 3.9 & 0.709 \\
\hline Repeat hospitalization (n, \%) & 5 & 0.3 & 6 & 0.4 & 0.763 \\
\hline Mean [SD] hospitalizations per month & 0.01 & {$[0.05]$} & 0.01 & {$[0.05]$} & 0.813 \\
\hline Mean [SD] length of stay, hospitalizations & 4.23 & {$[3.17]$} & 5.39 & {$[5.14]$} & $<0.001$ \\
\hline Office visit, any (n, \%) & 904 & 60.8 & 929 & 62.5 & 0.346 \\
\hline Mean [SD] office visits per month & 0.26 & {$[0.43]$} & 0.25 & {$[0.38]$} & 0.846 \\
\hline ER visit, any (n, \%) & 69 & 4.6 & 74 & 5.0 & 0.668 \\
\hline Mean [SD] ER visits per month & 0.03 & {$[0.26]$} & 0.04 & {$[0.24]$} & 0.877 \\
\hline \multicolumn{6}{|l|}{ Mean [SD] gout-specific cost per month, \$ } \\
\hline Hospitalization, any & 20 & [269] & 24 & [198] & 0.688 \\
\hline Repeat hospitalization & 113 & [626] & 131 & [449] & 0.372 \\
\hline Office visit, any & 11 & [29] & 12 & [23] & 0.786 \\
\hline Repeat office visits & 19 & [35] & 19 & [26] & 0.936 \\
\hline ER visit, any & 2 & [22] & 3 & {$[22]$} & 0.833 \\
\hline Repeat ER visits & 53 & [90] & 53 & [83] & 0.959 \\
\hline Prescription drug & 33 & [72] & 195 & [132] & $<0.001$ \\
\hline Allopurinol & 5 & [3] & NA & NA & NA \\
\hline Febuxostat & NA & NA & 168 & {$[105]$} & NA \\
\hline Other gout-specific prescription drugs & 28 & [71] & 27 & [63] & 0.912 \\
\hline Total costs & 87 & [389] & 243 & [265] & $<0.001$ \\
\hline
\end{tabular}

$(P=0.001)$. Unadjusted inpatient cost per month was lower $(P=0.465)$ in the febuxostat cohort ( $\$ 397$ vs. $\$ 489)$, but these gains were offset by significantly higher outpatient pharmacy cost in the febuxostat cohort $(P<0.001)$.

Mean (SD) expenditure per month for gout-specific services was $\$ 243$ (\$265) and $\$ 87$ (\$389) in the febuxostat and allopurinol cohorts, respectively $(P<0.001)$. Eighty percent of goutspecific cost in the febuxostat cohort was driven by gout-specific outpatient pharmacy cost (\$195 vs. \$33 in the allopurinol cohort, $P<0.001$ ), namely the cost of the comparator agents (Table 3).

Mean (SD) expenditure per month for all non-gout-specific services, unadjusted, was $\$ 1,282(\$ 3,820)$ in the febuxostat cohort and $\$ 1,403(\$ 3,861)$ in the allopurinol cohort $(P=0.389)$. Table 4 provides utilization detail for each of the measured non-gout conditions. Patients in the febuxostat cohort had marginally $(P=0.065)$ fewer renal hospitalizations per month and significantly fewer repeat hospitalizations $(P=0.036)$, but significantly longer lengths of stay $(P=0.001)$. Febuxostat patients were also significantly $(P=0.002)$ less likely to have had renal-related emergency room visits and had significantly $(P=0.048)$ lower renal outpatient cost. Similarly, patients in the febuxostat cohort had significantly fewer cardiovascular hospitalizations per month $(P=0.022)$ and repeat hospitalizations $(P=0.017)$, but longer lengths of stay $(P<0.001)$. The cost of cardiovascular-related office visits $(P=0.003)$ as well as over- all outpatient cardiovascular cost $(P=0.038)$ was significantly lower, unadjusted, in the febuxostat cohort.

Multivariate Analyses. Multivariate analysis was required because the post-match cohorts were not balanced in terms of line of ULT. GLM results (Table 5) suggest that febuxostat users treated in the second-line setting $(n=743)$ incurred significantly $(P=0.001)$ more total cost per patient per month than allopurinol users $(n=1,486)$ treated primarily in the first-line setting. Conversely, febuxostat users treated in the first-line setting $(n=743)$ incurred significantly $(P=0.009)$ less total cost than allopurinol users treated almost exclusively (95.8\%) in the same setting.

Multivariate models also found that febuxostat users in both settings had significantly $(P<0.001)$ higher gout-specific cost, due almost entirely to higher febuxostat acquisition cost. Increased gout-specific expenditure in the first-line febuxostat cohort was offset by significantly lower cardiovascular and renal-related costs $(P<0.001)$ and marginally lower diabetesrelated cost $(P=0.060)$. There were no significant differences in renal, cardiovascular, or diabetes-related costs between the allopurinol cohort and febuxostat patients treated in the secondline setting. Sensitivity analyses employing alternate distributional assumptions (Wald) in those models with ambiguous Park test coefficients were very similar to those produced in the 
TABLE 4 Non-Gout-Related Utilization in the Post-match Sample, Unadjusted

\begin{tabular}{|c|c|c|c|c|c|}
\hline & \multicolumn{2}{|c|}{$\begin{array}{c}\text { Allopurinol } \\
(\mathrm{n}=1,486)\end{array}$} & \multicolumn{2}{|c|}{$\begin{array}{c}\text { Febuxostat } \\
(\mathrm{n}=1,486)\end{array}$} & $P$ Value \\
\hline \multicolumn{6}{|l|}{ Renal-specific } \\
\hline Hospitalization, any (n, \%) & 244 & 16.4 & 208 & 14.0 & 0.066 \\
\hline Repeat hospitalization (n, \%) & 42 & 2.8 & 25 & 1.7 & 0.036 \\
\hline Mean [SD] hospitalizations per month & 0.03 & {$[0.12]$} & 0.03 & {$[0.09]$} & 0.065 \\
\hline Mean [SD] length of stay, days & 5.12 & {$[4.86]$} & 5.71 & {$[4.92]$} & 0.001 \\
\hline Office visit, any (n, \%) & 1,065 & 71.7 & 1,065 & 71.7 & 1.000 \\
\hline Mean [SD] office visits per month & 0.31 & {$[0.39]$} & 0.29 & {$[0.36]$} & 0.233 \\
\hline ER visit, any (n, \%) & 95 & 6.4 & 57 & 3.8 & 0.002 \\
\hline Mean [SD] ER visits per month & 0.04 & {$[0.45]$} & 0.02 & {$[0.16]$} & 0.116 \\
\hline \multicolumn{6}{|l|}{ Cardiovascular-specific } \\
\hline Hospitalization, any (n, \%) & 298 & 20.1 & 274 & 18.4 & 0.264 \\
\hline Repeat hospitalization (n, \%) & 75 & 5.0 & 49 & 3.3 & 0.017 \\
\hline Mean [SD] hospitalizations per month & 0.04 & {$[0.13]$} & 0.03 & {$[0.10]$} & 0.022 \\
\hline Mean [SD] length of stay, days & 4.92 & {$[4.70]$} & 5.72 & {$[5.82]$} & $<0.001$ \\
\hline Office visit, any $(n, \%)$ & 1,109 & 74.6 & 1,096 & 73.8 & 0.586 \\
\hline Mean [SD] office visits per month & 0.47 & {$[0.61]$} & 0.44 & {$[0.57]$} & 0.124 \\
\hline ER visit, any (n, \%) & 264 & 17.8 & 231 & 15.5 & 0.104 \\
\hline Mean [SD] ER visits per month & 0.18 & {$[0.85]$} & 0.14 & {$[0.78]$} & 0.134 \\
\hline Prescription drug use (n, \%) & 1,410 & 94.9 & 1,391 & 93.6 & 0.135 \\
\hline \multicolumn{6}{|l|}{ Diabetes-specific } \\
\hline Hospitalization, any (n, \%) & 99 & 6.7 & 89 & 6.0 & 0.451 \\
\hline Repeat hospitalization (n, \%) & 24 & 1.6 & 10 & 0.7 & 0.016 \\
\hline Mean [SD] hospitalizations per month & 0.02 & {$[0.09]$} & 0.01 & {$[0.06]$} & 0.069 \\
\hline Mean [SD] length of stay, days & 4.21 & {$[3.57]$} & 6.03 & {$[7.63]$} & $<0.001$ \\
\hline Office visit, any (n, \%) & 517 & 34.8 & 528 & 35.5 & 0.673 \\
\hline Mean [SD] office visits per month & 0.19 & {$[0.40]$} & 0.18 & {$[0.39]$} & 0.808 \\
\hline ER visit, any (n, \%) & 90 & 6.1 & 78 & 5.2 & 0.341 \\
\hline Mean [SD] ER visits per month & 0.10 & {$[1.35]$} & 0.05 & {$[0.37]$} & 0.206 \\
\hline Prescription drug use (n, \%) & 560 & 37.7 & 554 & 37.3 & 0.820 \\
\hline \multicolumn{6}{|l|}{ Other non-gout-specific } \\
\hline Hospitalization, any (n, \%) & 23 & 1.5 & 19 & 1.3 & 0.534 \\
\hline Repeat hospitalization (n, \%) & 2 & 0.1 & 2 & 0.1 & 1.000 \\
\hline Mean [SD] hospitalizations per month & 0.00 & {$[0.02]$} & 0.00 & {$[0.02]$} & 0.956 \\
\hline Mean [SD] length of stay, days & 4.76 & {$[4.24]$} & 4.18 & {$[3.50]$} & $<0.001$ \\
\hline Office visit, any (n, \%) & 1,108 & 74.6 & 1,133 & 76.2 & 0.287 \\
\hline Mean [SD] office visits per month & 0.53 & {$[0.70]$} & 0.55 & {$[0.64]$} & 0.427 \\
\hline ER visit, any (n, \%) & 392 & 26.4 & 346 & 23.3 & 0.051 \\
\hline Mean [SD] ER visits per month & 0.37 & [1.43] & 0.28 & {$[1.08]$} & 0.040 \\
\hline Prescription drug use (n, \%) & 1,442 & 97.0 & 1,427 & 96.0 & 0.133 \\
\hline
\end{tabular}

gamma, log link models. Scaled deviance ranged from 1.0 to 1.3. Sensitivity analysis, which allocated cost based only on the primary diagnosis, shifted cost from disease-specific categories to "other" but did not otherwise alter study findings.

\section{Discussion}

To our knowledge, the current study is the first to compare economic outcomes among renally compromised gout patients in the year following initiation on the 2 most common ULTs, allopurinol and febuxostat. This study demonstrated that in the first-line setting, gout patients with concurrent CKD treated with febuxostat incurred significantly less total cost than patients treated with allopurinol, despite the higher drug acquisition cost of febuxostat. Higher gout-specific expenditure was offset by lower cardiovascular $(P<0.001)$, renal $(P<0.001)$, and diabetes-related $(P=0.060)$ costs. In addition, patients treated with second-line febuxostat following allopurinol incurred significantly higher total cost than first-line allopurinol users $(P=0.001)$. This difference may be due to the presence of more aggressive disease from delayed effective 


\begin{tabular}{|c|c|c|c|c|c|c|}
\hline & \multirow{2}{*}{\multicolumn{2}{|c|}{$\begin{array}{c}\text { Allopurinol }^{\mathrm{a}} \\
(\mathrm{n}=1,486)\end{array}$}} & \multicolumn{4}{|c|}{ Febuxostat } \\
\hline & & & \multicolumn{2}{|c|}{ First-Line Setting $(\mathrm{n}=743)$} & \multicolumn{2}{|c|}{ Second-Line Setting $(n=743)$} \\
\hline & Mean, \$ & $95 \% \mathrm{CI}, \$$ & Mean, \$ & $95 \%$ CI, \$ & Mean, \$ & $95 \% \mathrm{CI}, \$$ \\
\hline Total cost $\mathrm{t}^{\mathrm{b}}$ & 1,487 & $1,477-1,498$ & 1,299 & $1,287-1,311^{\mathrm{c}}$ & 1,751 & $1,733-1,769^{d}$ \\
\hline Gout-specific cost & 87 & $86-87$ & 243 & $243-244 \mathrm{e}$ & 242 & $241-243^{e}$ \\
\hline \multicolumn{7}{|l|}{ Non-gout-specific cost } \\
\hline Renal & 216 & $212-219$ & 86 & $84-88^{e}$ & 245 & $241-252$ \\
\hline Cardiovascular & 459 & $455-463$ & 288 & $284-291^{\mathrm{e}}$ & 476 & $470-482$ \\
\hline Diabetes & 179 & $178-180$ & 151 & $150-152^{\mathrm{f}}$ & 183 & $182-184$ \\
\hline \multicolumn{7}{|l|}{$\begin{array}{l}\text { a Allopurinol is the referen } \\
{ }^{b} \text { Total cost is reported in } 2 \\
{ }^{c} P=0.0091 . \\
{ }^{d} P=0.0013 . \\
{ }^{e} P<0.0001 . \\
f_{P}=0.0622 . \\
C I=\text { confidence interval. }\end{array}$} \\
\hline
\end{tabular}

ULT, given that these patients had previously failed treatment with allopurinol. Patients with concurrent gout and CKD represent a clinically complex and resource-intensive population requiring management of both gout- and non-gout-related conditions. Accordingly, the cost impact of the decision to treat with allopurinol or febuxostat is likely more complex than a comparison of ULT drug acquisition cost alone.

Total, all-cause, and gout-specific cost in patients with gout has been reported in several studies. In the current study, total cost per month $(\$ 1,507)$ and gout-specific cost $(\$ 165)$ per month were slightly higher than results from studies conducted in a broad cross-section of patients, ${ }^{11,21,43}$ but more closely aligned with studies conducted in more highly comorbid populations. Wu et al. (2008) reported total annual cost among gout patients $\geq 65$ years at $\$ 14,734$ ( $\$ 1,228$ per month) and goutspecific cost at $\$ 876$ (\$73 per month). ${ }^{14}$ In a separate study, Wu et al. (2012) reported total cost among patients with 6 or more flares per year at $\$ 25,778(\$ 2,148$ per month) and gout-specific cost at $\$ 12,620$ per year ( $\$ 1,052$ per month). ${ }^{15}$

Kim et al. described clinical characteristics and health care utilizations of gout patients in a large U.S. populationbased cohort initiating allopurinol, febuxostat, or colchicine. ${ }^{41}$ This study found that febuxostat initiators were highly comorbid, had twice the rate of CKD, and had greater use of medications and health care resources than those initiating allopurinol or colchicine. Febuxostat initiators also had the highest incidence of acute gout flares, which the authors acknowledge may be due to the tendency to treat more severe gout with febuxostat.

In the United Kingdom, a cost-effectiveness study by Beard et al. $(2014)^{28}$ concluded that second-line treatment with febuxostat $80 \mathrm{mg} / 120 \mathrm{mg}$ is cost-effective following allopurinol $300 \mathrm{mg}$, even when accounting for mild-to-moderate renal impairment and titration of allopurinol. The Beard study differed from the current study with respect to the cost outcome (gout-related costs vs. total direct medical expenditure), study design (Markov health-state model vs. retrospective observational cohort study), and study population (all gout patients and a subgroup of patients with mild-to-moderate renal impairment vs. gout patients with CKD stage 3 or 4). Despite these differences, the current study came to the same conclusion as Beard et al.; first-line treatment with allopurinol results in lower gout-related costs. However, the Beard study did not examine cost impact beyond gout-related costs, whereas the current study demonstrated that the higher gout-related costs with first-line febuxostat were offset by significantly lower cardiovascular and renal-related costs in a real-world setting.

Gandhi et al. (2015) $)^{44}$ recently published a Markov model of clinical trial data, comparing direct health care costs for febuxostat and allopurinol from a U.S. payer's perspective. Over a 5-year period, the expected treatment success for febuxostat was $72 \%$, with an expected success rate of $42 \%$ for allopurinol. The authors found an incremental cost-effectiveness ratio of $\$ 6,322$ (in 2014 U.S. dollars) per treatment success for febuxostat, leading the authors to conclude that febuxostat was cost-effective from a U.S. payer's perspective. The Gandhi study differed from the present study with respect to study design (Markov health-state model vs. retrospective observational cohort study) and study population (all gout patients and a subgroup of patients with mild-to-moderate renal impairment vs. gout patients with CKD stage 3 or 4). Jutkowitz et al. (2014) also used Markov modeling to assess the lifetime cost impact of 5 urate-lowering treatment strategies in a cross-section of gout patients..$^{45}$ The authors concluded that allopurinol as a single therapy option is cost saving compared with no treatment, and that dose escalation of allopurinol-febuxostat sequential 
therapy is also cost-effective. Like the Gandhi study, this study used the literature to construct a theoretical model in a broad cross-section of patients. Neither of these models focused on the subset of patients with moderate-to-severe CKD.

The results of the current study have important implications for the management of gout in a renally challenged population. Despite considerable evidence that gout is a disease with suboptimal management and low adherence to treatment, ${ }^{46}$ there remains a lack of consensus on the optimal management of gout patients with renal disease. In 2012, the American College of Rheumatology (ACR) released guidelines that included recommendations that CKD patients (stage 2-4) be considered candidates for ULT, with either allopurinol or febuxostat as the first-line agent. ${ }^{2}$ Although the ACR guidelines specify that allopurinol hypersensitivity syndrome can be minimized by dose titration in patients with renal impairment, ${ }^{2}$ these guidelines do not contain specific recommendations on use of ULTs in renally impaired patients. ${ }^{46}$ In contrast, other guidelines recommend only allopurinol as the first-line ULT, citing economic models in the United Kingdom that show the cost-effectiveness of febuxostat in the general population only in second-line settings ${ }^{46}$ Choice of first-line ULT may also be influenced by the potential risk of cardiovascular events with use of febuxostat. According to guidance from the National Institute for Health and Care Excellence (NICE) ${ }^{47}$ febuxostat is not recommended for people with ischemic heart disease or congestive heart failure. The U.S. Food and Drug Administration (FDA) label ${ }^{48}$ includes a warning to monitor for signs of myocardial infarction and stroke.

A growing number of clinicians have concluded that hyperuricemia is an independent cardiovascular risk factor. ${ }^{30,31,33,34,36,49,50}$ Systematic reviews have demonstrated significant relationships between gout or elevated sUA and incident coronary artery disease, CVD-related mortality, ${ }^{51-54}$ stroke incidence, and mortality. ${ }^{55}$ In addition, studies have suggested sUA as a strong independent prognostic factor in patients with heart failure ${ }^{56,57}$ and determined that a high-normal sUA level is associated with worse ejection fraction in heart failure patients, impaired stroke volume and cardiac output, cognitive function, and arterial stiffness in overall healthy adult-elderly subjects. ${ }^{58}$ Another recent meta-analysis demonstrated a linear association between sUA level and mortality: elevated sUA increased the risk of all-cause mortality (relative risk $[R R]=1.24,95 \%$ confidence interval $[\mathrm{CI}]=1.09-1.42$ ) and cardiovascular mortality $(\mathrm{RR}=1.37,95 \% \mathrm{CI}=1.19-1.57) .^{59}$ In studies of urate-lowering medications, control of sUA was associated with a reduction in cardiovascular risk, but it is not known if this is due to direct effects of lowered sUA or to other benefits from these medications such as reduced oxidative stress and other pleiotropic effects on the endothelium. ${ }^{39}$
This possibility underlines the importance of appropriate gout management, especially in patients with comorbid conditions. Although the present study did not analyze the impact of gout treatment on comorbid conditions clinically, the findings did show a beneficial impact of first-line febuxostat on the cost of cardiovascular and renal comorbid conditions. Although this finding seems to contradict the small numerically higher rate of cardiovascular thromboembolic events observed in patients treated with febuxostat in clinical trials (as noted in the FDA and NICE product guidance), it is important to note that this increased rate was not statistically significant and there is no evidence to date of a causal relationship between febuxostat and cardiovascular events. ${ }^{60}$ In the current study, it is not clear why cardiovascular and renal-related costs were lower with febuxostat. Although we presume cost reductions reflect the favorable impact of lower sUA on cardiovascular events, unmeasured confounding may exist. Further studies are warranted to examine the relationship between cardiovascular events and use of febuxostat.

\section{Limitations}

Although the current study was based on a sufficient sample of patients with gout and CKD, it is not a random sample and the sources of the data are worth reviewing. The MarketScan databases represent patients with either commercial or Medicare supplemental insurance. As such, study findings may not be generalizable to other populations such as the uninsured or patients with other coverage, such as Medicaid.

These data are collected to facilitate reimbursement and as such do not contain all relevant socioeconomic and clinical data. Medical records were not available to supplement or validate CKD stage, comorbidity may have been underreported, measures of alcohol and tobacco use were absent, and sUA results were unavailable. Medication exposure may have been under- or overstated because prescription medications were presumed to be taken based on their pharmacy fill dates with coverage windows based on days supply. Moreover, sample size limitations prevented propensity matching the first-line and second-line treatment groups separately, primarily because of the limited use of allopurinol in a second-line setting. Postmatch multivariate modeling was used to address the residual imbalance.

Finally, it is also conceivable that clinicians are cautious in using febuxostat in patients with existing CVD and therefore self-select patients with comparatively mild or stable disease. Similarly, it is conceivable that clinicians are also cautious in using allopurinol in patients with renal disease so that they self-select patients they perceive as having more stable disease. Although the current study used multiple methods to address such selection bias, some degree of unmeasured confounding may remain. 


\section{Conclusions}

Gout patients with concurrent CKD who initiated treatment with febuxostat in a first-line setting incurred significantly less total cost than patients initiating allopurinol during the first exposure to each agent. Conversely, patients treated with second-line febuxostat following allopurinol incurred significantly higher total cost than patients initiating allopurinol. There was no significant difference in total cost between the agents across line of therapy.

Although study findings suggest the potential for cardiovascular- and renal-related savings to offset febuxostat's higher acquisition in gout patients with moderate-to-severe CKD, this is the first such retrospective evaluation. Future research is warranted to both demonstrate the durability of study findings and to better elucidate the mechanism by which associated cost offsets occur.

\section{Authors}

GHAITH MITRI, MD, is Medical Director, Mid-America Region, naviHealth, Chicago, Illinois; ERIC T. WITTBRODT, PharmD, MPH, is Principal Health Outcomes Liaison, Daiichi Sankyo, Parsipanny, New Jersey; and ROBIN S. TURPIN, PhD, is Director and Head, U.S. Health Economics and Outcomes Research, Medical Affairs, Takeda Pharmaceuticals U.S.A., Deerfield, Illinois. BENI A. TIDWELL, BS, is Research Associate, and KATHY L. SCHULMAN, MS, is Research Scientist and Principal, Outcomes Research Solutions, Shrewsbury, Massachusetts.

AUTHOR CORRESPONDENCE: Robin S. Turpin, PhD, Director and Head, U.S. HEOR, Medical Affairs, Takeda Pharmaceuticals U.S.A, One Takeda Pkwy., Deerfield, IL 60015. Tel.: 224.554.1730; Fax: 224.554.7916; E-mail: robin.turpin@takeda.com.

\section{DISCLOSURES}

No outside funding supported this study. Turpin is an employee of Takeda Pharmaceuticals U.S.A. Mitri and Wittbrodt were employees of Takeda Pharmaceuticals U.S.A. at the time of this study. Tidwell and Schulman are employees of Outcomes Research Solutions, consultants to Takeda Pharmaceuticals U.S.A.

All authors contributed to the design of the study and to the writing and review of the manuscript. All authors read and approved the final manuscript. Tidwell and Schulman collected the data, and all authors participated in data interpretation.

\section{ACKNOWLEDGMENTS}

The authors wish to thank Karina Berenson, MPH, for her assistance with manuscript preparation, and Laurie A. Costa, MPH, for SAS programming and analysis.

\section{REFERENCES}

1. Roddy E, Choi HK. Epidemiology of gout. Rheum Dis Clin North Am. 2014:40(2):155-75.
2. Khanna D, Fitzgerald JD, Khanna PP, et al. 2012 American College of Rheumatology guidelines for management of gout. Part 1: systematic nonpharmacologic and pharmacologic therapeutic approaches to hyperuricemia Arthritis Care Res (Hoboken). 2012;64(10):1431-46.

3. Lawrence RC, Felson DT, Helmick CG, et al. Estimates of the prevalence of arthritis and other rheumatic conditions in the United States. Part II. Arthritis Rheum. 2008;58(1):26-35.

4. Suresh E, Das P. Recent advances in management of gout. QJM. 2012;105(5):407-17.

5. Fravel MA, Ernst ME. Management of gout in the older adult. Am J Geriatr Pharmacother. 2011;9(5):271-85.

6. Juraschek SP, Kovell LC, Miller ER 3rd, Gelber AC. Association of kidney disease with prevalent gout in the United States in 1988-1994 and 2007-2010. Semin Arthritis Rheum. 2013;42(6):551-61.

7. Zhu Y, Pandya BJ, Choi HK. Comorbidities of gout and hyperuricemia in the U.S. general population: NHANES 2007-2008. Am J Med. 2012;125(7):679-87.

8. Khanna D, Khanna PP, Storgard C, Bumgartner S, Morlock R. Patients that continue to flare despite reaching Eular/ACR recommended serum urate target. Paper presented at: EULAR 2013, June 12-15, 2013; Madrid, Spain. Available at: http://ard.bmj.com/content/72/Suppl_3/A713.3.abstract. Accessed December 16, 2015.

9. Brook RA, Kleinman NL, Patel PA, et al. The economic burden of gout on an employed population. Curr Med Res Opin. 2006;22(7):1381-89.

10. Kim KY, Ralph Schumacher H, Hunsche E, Wertheimer AI, Kong SX. A literature review of the epidemiology and treatment of acute gout. Clin Ther 2003;25(6):1593-617

11. Park H, Rascati KL, Prasla K, McBayne T. Evaluation of health care costs and utilization patterns for patients with gout. Clin Ther. 2012;34(3):640-52.

12. Sicras-Mainar A, Navarro-Artieda R, Ibáñez-Nolla J. Resource use and economic impact of patients with gout: a multicenter, population-wide study. Reumatol Clin. 2013;9(2):94-100.

13. Trieste L, Palla I, Fusco F, et al. The economic impact of gout: a systematic literature review. Clin Exp Rheumatol. 2012;30(4 Suppl 73):S145-48

14. Wu EQ, Patel PA, Yu AP, et al. Disease-related and all-cause health care costs of elderly patients with gout. J Manag Care Pharm. 2008;14(2):164-75. Available at: http://www.amcp.org/data/jmcp/JMCPMaga_March\%2008_164-175.pdf.

15. Wu EQ, Forsythe A, Guerin A, Yu AP, Latremouille-Viau D, Tsaneva M. Comorbidity burden, healthcare resource utilization, and costs in chronic gout patients refractory to conventional urate-lowering therapy. Am J Ther. 2012;19(6):e157-66

16. Wu EQ, Patel PA, Mody RR, et al. Frequency, risk, and cost of gout-related episodes among the elderly: does serum uric acid level matter? J Rheumatol. 2009;36(5):1032-40.

17. Roddy E, Zhang W, Doherty M. Is gout associated with reduced quality of life? A case-control study. Rheumatology (Oxford). 2007;46(9):1441-44.

18. Lee SJ, Hirsch JD, Terkeltaub R, et al. Perceptions of disease and healthrelated quality of life among patients with gout. Rheumatology (Oxford). 2009;48(5):582-86.

19. Singh JA, Strand V. Gout is associated with more comorbidities, poorer health-related quality of life and higher healthcare utilisation in U.S. veterans. Ann Rheum Dis. 2008;67(9):1310-16.

20. Singh JA. The impact of gout on patient's lives: a study of AfricanAmerican and Caucasian men and women with gout. Arthritis Res Ther. 2014;16(3):R132.

21. Lynch W, Chan W, Kleinman N, Andrews LM, Yadao AM. Economic burden of gouty arthritis attacks for employees with frequent and infrequent attacks. Popul Health Manag. 2013;16(2):138-45. 
22. Ye P, Yang S, Zhang W, et al. Efficacy and tolerability of febuxostat in hyperuricemic patients with or without gout: a systematic review and metaanalysis. Clin Ther. 2013;35(2):180-89.

23. Stamp LK, Jordan S. The challenges of gout management in the elderly. Drugs Aging. 2011;28(8):591-603.

24. Sarawate CA, Patel PA, Schumacher HR, Yang W, Brewer KK, Bakst AW. Serum urate levels and gout flares: analysis from managed care data. J Clin Rheumatol. 2006;12(2):61-65.

25. Becker MA, Schumacher HR, Espinoza LR, et al. The urate-lowering efficacy and safety of febuxostat in the treatment of the hyperuricemia of gout: the CONFIRMS trial. Arthritis Res Ther. 2010;12(2):R63.

26. Becker MA, Schumacher HR Jr., Wortmann RL, et al. Febuxostat compared with allopurinol in patients with hyperuricemia and gout. N Engl J Med. 2005;353(23):2450-61.

27. Schumacher HR Jr., Becker MA, Wortmann RL, et al. Effects of febuxostat versus allopurinol and placebo in reducing serum urate in subjects with hyperuricemia and gout: a 28-week, phase III, randomized, double-blind, parallel-group trial. Arthritis Rheum. 2008;59(11):1540-48.

28. Beard SM, von Scheele BG, Nuki G, Pearson IV. Cost-effectiveness of febuxostat in chronic gout. Eur J Health Econ. 2014;15(5):453-63.

29. Gray CL, Walters-Smith NE. Febuxostat for treatment of chronic gout. Am J Health Syst Pharm. 2011;68(5):389-98.

30. Richette P, Perez-Ruiz F, Doherty M, et al. Improving cardiovascular and renal outcomes in gout: what should we target? Nat Rev Rheumatol. 2014;10(11):654-61.

31. Perez-Ruiz F, Dalbeth N, Bardin T. A review of uric acid, crystal deposition disease, and gout. Adv Ther. 2015;32(1):31-41.

32. Kanbay M, Yilmaz MI, Sonmez A, et al. Serum uric acid independently predicts cardiovascular events in advanced nephropathy. Am J Nephrol. 2012;36(4):324-31.

33. Kanbay M, Segal M, Afsar B, Kang DH, Rodriguez-Iturbe B, Johnson RJ. The role of uric acid in the pathogenesis of human cardiovascular disease. Heart. 2013;99(11):759-66.

34. Grassi D, Desideri G, Di Giacomantonio AV, Di Giosia P, Ferri C. Hyperuricemia and cardiovascular risk. High Blood Press Cardiovasc Prev. 2014:21(4):235-42.

35. Neogi T. Asymptomatic hyperuricemia: perhaps not so benign? J Rheumatol. 2008;35(5):734-37.

36. Soltani Z, Rasheed K, Kapusta DR, Reisin E. Potential role of uric acid in metabolic syndrome, hypertension, kidney injury, and cardiovascular diseases: is it time for reappraisal? Curr Hypertens Rep. 2013;15(3):175-81.

37. Austin PC. Balance diagnostics for comparing the distribution of baseline covariates between treatment groups in propensity-score matched samples. Stat Med. 2009;28(25):3083-107.

38. Austin PC. The relative ability of different propensity score methods to balance measured covariates between treated and untreated subjects in observational studies. Med Decis Making. 2009;29(6):661-77.

39. Austin PC. Goodness-of-fit diagnostics for the propensity score model when estimating treatment effects using covariate adjustment with the propensity score. Pharmacoepidemiol Drug Saf. 2008;17(12):1202-17.

40. Austin PC. A critical appraisal of propensity-score matching in the medical literature between 1996 and 2003. Stat Med. 2008;27(12):2037-49.

41. Kim SC, Schmidt BM, Franklin JM, Liu J, Solomon DH, Schneeweiss S. Clinical and health care use characteristics of patients newly prescribed allopurinol, febuxostat and colchicine for gout. Arthritis Care Res (Hoboken). 2013;65(12):2008-14.

42. D'Agostino RB Jr. Propensity score methods for bias reduction in the comparison of a treatment to a non-randomized control group. Stat Med. 1998;17(19):2265-81.
43. Saseen JJ, Agashivala N, Allen RR, Ghushchyan V, Yadao AM, Nair KV. Comparison of patient characteristics and gout-related health-care resource utilization and costs in patients with frequent versus infrequent gouty arthritis attacks. Rheumatology (Oxford). 2012;51(11):2004-12.

44. Gandhi PK, Gentry WM, Ma Q, Bottorff MB. Cost-effectiveness analysis of allopurinol versus febuxostat in chronic gout patients: a U.S. payer perspective. J Manag Care Spec Pharm. 2015;21(2):165-75. Available at: http:// www.jmcp.org/doi/abs/10.18553/jmcp.2015.21.2.165.

45. Jutkowitz E, Choi HK, Pizzi LT, Kuntz KM. Cost-effectiveness of allopurinol and febuxostat for the management of gout. Ann Intern Med. 2014;161(9):617-26.

46. Nuki G. An appraisal of the 2012 American College of Rheumatology Guidelines for the Management of Gout. Curr Opin Rheumatol. 2014;26(2):152-61.

47. National Institute for Health and Care Excellence. Febuxostat for the management of hyperuricaemia in people with gout. 2008. Available at: http://www.nice.org.uk/guidance/tal64. Accessed December 16, 2015.

48. ULORIC (febuxostat) tablet for oral use. Takeda Pharmaceuticals America. Revised March 2013. Available at: http://general.takedapharm.com/ content/file.aspx?applicationcode $=66$ b0b942-e82b-46ad-886a-f4aa59f5f33 c\&filetypecode=ULORICPI\&cacheRandomizer=dlcb0b9f-494e-4e9e-994aedbb3f66d544. Accessed January 7, 2016.

49. Neogi T. Asymptomatic hyperuricemia: cardiovascular and renal complications. In: Terkeltaub R, ed. Gout and Other Crystal Arthropathies. Philadelphia: Elsevier Saunders; 2012:226-38.

50. Stamp LK, Chapman PT. Gout and its comorbidities: implications for therapy. Rheumatology (Oxford). 2013;52(1):34-44.

51. Clarson L, Chandratre P, Hider SL, et al. Increased cardiovascular mortality associated with gout: a systematic review and meta-analysis. Eur J Prev Cardiol. 2015;22(3):335-43.

52. Clarson LE, Hider SL, Belcher J, Heneghan C, Roddy E, Mallen CD. Increased risk of vascular disease associated with gout: a retrospective, matched cohort study in the UK clinical practice research datalink. Ann Rheum Dis. 2015;74(4):642-47.

53. Lottmann K, Chen X, Schädlich PK. Association between gout and all-cause as well as cardiovascular mortality: a systematic review. Curr Rheumatol Rep. 2012;14(2):195-203.

54. Kim SY, Guevara JP, Kim KM, Choi HK, Heitjan DF, Albert DA. Hyperuricemia and coronary heart disease: a systematic review and metaanalysis. Arthritis Care Res. 2010;62(2):170-80.

55. Kim SY, Guevara JP, Kim KM, Choi HK, Heitjan DF, Albert DA. Hyperuricemia and risk of stroke: a systematic review and meta-analysis. Arthritis Rheum. 2009;61(7):885-92.

56. Bergamini C, Cicoira M, Rossi A, Vassanelli C. Oxidative stress and hyperuricaemia: pathophysiology, clinical relevance, and therapeutic implications in chronic heart failure. Eur J Heart Fail. 2009;11(5):444-52.

57. Huang H, Huang B, Li Y, et al. Uric acid and risk of heart failure: a systematic review and meta-analysis. Eur J Heart Fail. 2014;16(1):15-24.

58. Borghi C, Verardi FM, Pareo I, Bentivenga C, Cicero AF. Hyperuricemia and cardiovascular disease risk. Expert Rev Cardiovasc Ther. 2014;12(10):1219-25.

59. Zhao G, Huang L, Song M, Song Y. Baseline serum uric acid level as a predictor of cardiovascular disease related mortality and all-cause mortality: a meta-analysis of prospective studies. Atherosclerosis. 2013;231(1):61-68.

60. Garcia-Valladares I, Khan T, Espinoza LR. Efficacy and safety of febuxostat in patients with hyperuricemia and gout. Ther Adv Musculoskelet Dis. 2011;3(5):245-53. 


\section{APPENDIX ICD-9-CM Diagnosis Code Definitions of Disease States}

\begin{tabular}{|c|c|c|c|}
\hline Disease State & ICD-9-CM Code & Description & Pharmacy/Procedures \\
\hline Gout & $274 . x x$ & Gout & $\begin{array}{l}\text { Allopurinol, febuxostat } \\
\text { Colchicine, probenecid, glucocorticoids } \\
\text { Prescription NSAIDs, sulfinpyrazone } \\
\text { Intra-articular aspiration and/or injection } \\
\text { (CPT codes } 20600,20605,20610 \text { ) } \\
\text { Examination musculoskeletal system/joint fluid } \\
\text { (ICD-9-CM procedure code } 91.5 \text { or CPT code } \\
89060 \text { ) } \\
\text { Uric acid estimation, blood and other (CPT } 84550 \text {, } \\
84560 \text { ) }\end{array}$ \\
\hline Cardiovascular & $\begin{array}{l}390-398 \\
401-405 \\
410-414 \\
415-417 \\
420-429 \\
430-438 \\
440-449 \\
451 . x x \\
452 \cdot x x \\
453 . x x \\
457 . x x \\
458 \cdot x x \\
459 . x x\end{array}$ & $\begin{array}{l}\text { Acute rheumatic fever \& chronic rheumatic heart } \\
\text { disease } \\
\text { Hypertensive disease } \\
\text { Ischemic heart disease } \\
\text { Diseases of pulmonary circulation } \\
\text { Other forms of heart disease } \\
\text { Cerebrovascular disease } \\
\text { Diseases of arteries, arterioles, capillaries } \\
\text { Phlebitis and thrombophlebitis } \\
\text { Portal vein thrombosis } \\
\text { Other venous embolism, thrombosis } \\
\text { Noninfectious dislymphatic channels } \\
\text { Hypotension } \\
\text { Other disorders of circulatory system }\end{array}$ & $\begin{array}{l}\text { Antiarrhythmic agents } \\
\text { Anticoagulants } \\
\text { Hemorrheologic agents } \\
\text { Sclerosing agents } \\
\text { Thrombolytics } \\
\text { Angiotensin-converting enzyme inhibitors } \\
\text { Angiotensin II receptor blockers } \\
\text { Antiplatelets } \\
\text { Beta-blockers } \\
\text { Calcium channel blockers } \\
\text { Digitalis preparations } \\
\text { Diuretics } \\
\text { Vasodilators }\end{array}$ \\
\hline Diabetes & $\begin{array}{l}250 . x x \\
357.2 \\
362.0 x \\
366.41 \\
\end{array}$ & $\begin{array}{l}\text { Diabetes mellitus } \\
\text { Polyneuropathy in diabetes } \\
\text { Diabetic retinopathy } \\
\text { Diabetic cataract }\end{array}$ & All oral or injectable antidiabetic agents \\
\hline Renal & $\begin{array}{l}\text { 580-589 } \\
\text { 590.xx } \\
\text { 591.xx } \\
\text { 592.xx } \\
\text { 593.xx } \\
\end{array}$ & $\begin{array}{l}\text { Nephritis, nephrotic syndrome, nephrosis } \\
\text { Infections of kidney } \\
\text { Hydronephrosis } \\
\text { Calculus of kidney and ureter } \\
\text { Other disorders of kidney and ureter }\end{array}$ & N/A \\
\hline
\end{tabular}

CPT = Current Procedural Terminology; ICD-9-CM = International Classification of Diseases, Ninth Revision, Clinical Modification; NSAID = nonsteroidal anti-inflammatory drug. 